\title{
Rapid detection of carbapenemase activity of Enterobacteriaceae isolated from positive blood cultures by MALDI-TOF MS
}

Jiajia Yu, Jingxian Liu, Yuanrui Li, Jing Yu, Weinan Zhu, Ying Liu* and Lisong Shen*

\begin{abstract}
Background: Matrix-assisted laser desorption ionization-time of flight mass spectrometry (MALDI-TOF MS) has been proved to be a useful tool for identification of pathogens directly isolated from blood cultures in clinical microbiology laboratories. $\beta$-Lactam antibiotics are commonly used for treatment of bloodstream infections caused by Enterobacteriaceae strains, and carbapenem is the superlative class of $\beta$-lactam antibiotics. Since the carbapenem resistance rate of Enterobacteriaceae strains raised year by year, efficient detection of carbapenemase activity and timely delivery of carbapenem susceptibility reports of Enterobacteriaceae strains isolated from blood cultures is important for clinicians.

Methods: We used 64 simulated blood cultures to establish the method of MALDI-TOF MS based ertapenem hydrolysis assay. The cutoff value of logRQ calculated from the peaks intensity of ertapenem and its hydrolysate was first set to identify the strains with carbapenemase activity. Then, we detected and calculated the logRQ values of 385 Enterobacteriaceae strains from positive clinical blood cultures to distinguish the carbapenemase producers and noncarbapenemase producers.

Results: The mean logRQ value of 32 noncarbapenemase producers was $-0.85 \pm 0.14$ in simulated blood cultures, while the logRQ value of 32 carbapenemase producers was $0.87 \pm 0.55$. Thus, the cutoff value of logRQ was set at -0.45 with sensitivity of $100 \%$ and specificity of $100 \%$. In 385 clinical positive blood cultures, the logRQ values of all carbapenem-susceptible Enterobacteriaceae strains $(81.3 \%, 313 / 385)$ were $<-0.45$. Comparing with the detection of carbapenemase genes, carbapenem-resistant Enterobacteriaceae strains (18.7\%, 72/385) were well distinguished by MALDI-TOF MS based ertapenem hydrolysis assay with a sensitivity of $92.5 \%$ and specificity of $100 \%$.

Conclusions: Our data show that MALDI-TOF MS based ertapenem hydrolysis assay is a rapid and accurate method to detect carbapenemase activity of Enterobacteriaceae strains from positive blood cultures, and can be routinely performed in clinical microbiology laboratories.
\end{abstract}

Keywords: MALDI-TOF MS, Blood culture, Carbapenemase, Enterobacteriaceae, Hydrolysis

\section{Background}

Bloodstream infection is a severe threat for human life. As reported before, a mean survival rate of $7.6 \%$ was decreased if the effective treatment was applied per hour delay for patients in septic shock [1]. Therefore, early appropriate antibiotic treatment is important to improve

\footnotetext{
*Correspondence: liuying01@xinhuamed.com.cn; lisongshen@hotmail. com

Department of Clinical Laboratory, Xin Hua Hospital, Shanghai Jiao Tong University School of Medicine, 1665 Kong Jiang Road, Yangpu District, Shanghai 200092, China
}

prognosis of bloodstream infections [2]. Blood culture was considered as the 'gold standard' test for the detection of pathogens in blood specimens [3]. The rapid identification and susceptibility test reports for the pathogens in blood specimens are very meaningful for clinicians to choose appropriate and efficient antimicrobial agents for anti-infective therapy. Nowadays, Enterobacteriaceae strains are becoming the major pathogens which can cause bloodstream infections with an isolating rate up to $30 \%$ [4]. Carbapenem is the crucial antibacterial agent for primary empirical antibacterial therapy against infections

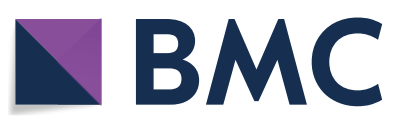

(c) The Author(s) 2018. This article is distributed under the terms of the Creative Commons Attribution 4.0 International License (http://creativecommons.org/licenses/by/4.0/), which permits unrestricted use, distribution, and reproduction in any medium, provided you give appropriate credit to the original author(s) and the source, provide a link to the Creative Commons license, and indicate if changes were made. The Creative Commons Public Domain Dedication waiver (http://creativecommons.org/ publicdomain/zero/1.0/) applies to the data made available in this article, unless otherwise stated. 
caused by Enterobacteriaceae strains. However, In the past few years, along with the large-scale use of antibiotics, carbapenem resistance of Enterobacteriaceae strains has increased rapidly and become one of the most common global health issues [5], especially for the carbapenem resistance rate of clinical isolated Klebsiella pneumoniae, which has reached up to $10 \%$ in 2014 [6, 7]. In addition, empirical antibacterial monotherapy may not well inhibit the growth of resistant Enterobacteriaceae strains [8]. Therefore, it's important for clinical microbiology laboratories to give clinicians timely and believable antimicrobial reports for early anti-infective treatment.

MALDI-TOF MS has been routinely used to identify microorganisms in clinical microbiology laboratories. Recently, it's reported that MALDI-TOF MS has been applied to the antimicrobial resistance research, especially for the activity detection of hydrolytic enzyme that produced by microorganisms. Here, we evaluated the efficiency of MALDI-TOF MS for detection the carbapenemase hydrolyze activity of Enterobacteriaceae strains isolated from positive blood cultures.

\section{Methods \\ Blood culture simulations}

64 Enterobacteriaceae strains were collected from patient specimens in the clinical microbiology laboratory of Xinhua Hospital, Shanghai JiaoTong University School of Medicine, Shanghai, China. The strains were constitute of 32 carbapenemase producers and 32 noncarbapenemase producers, and were stored in glycerol broth at $-80^{\circ} \mathrm{C}$. For each strain, after be inoculating and growing on Columbia blood plate at $35{ }^{\circ} \mathrm{C}$, bacterial suspensions of $0.5 \mathrm{McFarland}$ were prepared with $0.45 \% \mathrm{NaCl}$, and then diluted 100 times by $0.45 \% \mathrm{NaCl} .200 \mu \mathrm{L}$ of bacterial suspensions were injected into the negative blood culture bottles to simulate blood culture specimens. These blood culture bottles were incubated at $35{ }^{\circ} \mathrm{C}$ in the BD BACTEC FX Blood Culture System (Bectom Dickinson), and were taken out and detected by MALDI-TOF MS based ertapenem hydrolysis assay when the positive alerting signal was observed from the system.

\section{MALDI-TOF MS based ertapenem hydrolysis assay}

$4 \mathrm{~mL}$ samples from positive blood cultures were injected into serum separator tubes (ST740CG, INSEPACK ${ }^{\circledR}$, Japan), and centrifuged at $3000 \times g$ for $10 \mathrm{~min}$ in order to remove blood cells and debris at the bottom of tubes. The bacterial pellets at the surface of separation gel were carefully transferred into a $1.5 \mathrm{~mL}$ tube and washed twice using $500 \mu \mathrm{L} 0.45 \% \mathrm{NaCl}$. Then the pellets were resuspended with $50 \mu \mathrm{L}$ of ertapenem solution $\left(0.2 \mathrm{mg} \mathrm{mL}^{-1}\right.$ ertapenem, $20 \mathrm{mmol} \mathrm{L}^{-1}$ Tris- $\mathrm{HCl}, 0.45 \% \mathrm{NaCl}$ ) and then incubated at $35{ }^{\circ} \mathrm{C}$ for $2 \mathrm{~h}$. The ertapenem solution without any bacteria was set as the blank control. After $2 \mathrm{~h}$ incubation, the suspensions were centrifuged at $3000 \times g$ for $5 \mathrm{~min} .1 \mu \mathrm{L}$ of the supernatant was spotted on the target plate (Bruker Daltonics), subsequently mixed with $1 \mu \mathrm{L}$ HCCA matrix solution $\left(10 \mathrm{mg} \mathrm{mL}^{-1}\right.$ cyano-4-hydroxycinnamic acid) and then dried at room temperature. Mass spectrum was acquired by FlexControl 3.0 software (Bruker Daltonics) of Microflex LT mass spectrometer (Bruker Daltonics) in the low mass-tocharge range (100-1000) with $60 \mathrm{~Hz}$ laser frequency and 200 laser shots. The matrix peaks $\left(\mathrm{HCCA}[\mathrm{M}+\mathrm{H}]^{+}\right.$at 190.05 and $[2 \mathrm{M}+\mathrm{H}]^{+}$at 379.02 ) were used for calibration per test. Further analysis of mass spectrum was performed by the Flexanalysis 3.0 (Bruker Daltonics). Firstly, the spectrum was smoothed and baseline was subtracted. Then, the specific ertapenem related peaks and hydrolysate related peaks were manually checked in the massto-charge range of 440-560.

\section{LogRQ values}

The $\log R \mathrm{Q}$ values which were used to estimate the results of hydrolysis assay were calculated by the intensities of peaks. The formula was $\log R \mathrm{Q}=\log$ (sum of hydrolysis peak intensities)/(sum of ertapenem related peak intensities) $[9,10]$. Since both of the peaks of ertapenem and its hydrolysed, decarboxylated form may be observed in the mass spectrums of ertapenem solutions incubated with carbapenemase or non-carbapenemase producers, a cutoff value of $\log R Q$ was necessary to distinguish these two groups. The cutoff value was acquired based on receiver operating characteristic curve (ROC) which was conducted to determine the accuracy of the $\log R Q$ values to estimate the results of hydrolysis assay.

\section{Clinical blood cultures collection}

Patient positive blood culture bottles which contained Enterobacteriaceae strains were collected from October 2015 to December 2016 in the clinical microbiology laboratory of Xinhua Hospital. Both of aerobic and anaerobic blood culture bottles (BD BACTEC, Bectom Dickinson) from different body parts of patients were included. After blood culture bottles were accepted at our laboratory, they were put in the BD BACTEC FX Blood Culture System (Bectom Dickinson) immediately until the positive signals were obtained. The blood cultures incubated in blood culture system for 5 days with no positive signals were considered as negative. The bacteria from blood cultures bottles with positive signals were collected under centrifugal force in serum separator tubes and were placed onto the target plate for directly identification by MALDI-TOF MS. If the bacteria were finally 
identified as Enterobacteriaceae strains, their hydrolytic activity against ertapenem were further performed by MALDI-TOF MS according to "MALDI-TOF MS based ertapenem hydrolysis assay" that mentioned above. Klebsiella pneumoniae ATCC 700603 provided by Shanghai Clinical Laboratory Center was included as the negative control, while one Klebsiella pneumoniae KPC-2 gene positive strain which identified before in our laboratory was included as the positive control. The conventional inoculation, identification (Microflex LT, Bruker Daltonics) and antimicrobial susceptibility test (Vitek 2 Compact, BioMérieux) of pathogens isolated from positive blood cultures were performed at the same time. Ertapenem, meropenem and imipenem were used as carbapenem agents in the research. The strains were identified as carbapenem-resistant Enterobacteriaceae (CRE) if they were resistant to at least one of the three carbapenem agents.

\section{Molecular assay}

The carbapenemase genes, including $b l a_{K P C}, b l a_{I M P}$, $b l a_{N D M}, b l a_{V I M}, b l a_{G E S}$ and $b l a_{O X A-48}$ were detected by Polymerase Chain Reaction (PCR) and DNA sequencing within the strains which were indentified as CRE [11].

\section{Results}

\section{Strains for blood culture simulation}

The strains collected here covered 7 species of Enterobacteriaceae, including Klebsiella pneumoniae, Enterobacter cloacae, Enterobacter aerogenes, Escherichia coli, Citrobacter freundii, Klebsiella oxytoca and Raoultella ornithinolytica. 32 carbapenemase producers contain 6 kinds of carbapenemases in total, including KPC-2, NDM-1, IMP1, IMP-4, VIM-1 and VIM-2.

\section{Peaks of ertapenem and the hydrolysed, decarboxylated form}

Ertapenem intact peaks $[\mathrm{M}+\mathrm{H}]^{+}$at $476.5,[\mathrm{M}+\mathrm{Na}]^{+}$ at $498.5,[\mathrm{M}+\mathrm{K}]^{+}$at $514.5,[\mathrm{M}+2 \mathrm{Na}]^{+}$at 520.5 , $[\mathrm{M}+\mathrm{Na}+\mathrm{K}]^{+}$at 536.5 and $[\mathrm{M}+3 \mathrm{Na}]^{+}$at 542.5 and the peaks of their hydrolysed, decarboxylated form $\left[\mathrm{M}_{\text {hydr/decarb }}+\mathrm{H}\right]^{+}$at $450.5,\left[\mathrm{M}_{\text {hydr/decarb }}+\mathrm{Na}\right]^{+}$at 472.5 , $\left[\mathrm{M}_{\text {hydr/decarb }}+\mathrm{K}\right]^{+}$at $488.5,\left[\mathrm{M}_{\text {hydr }}+\mathrm{H}\right]^{+}$at 494.5 and $\left[\mathrm{M}_{\text {hydr }}+2 \mathrm{Na}\right]^{+}$at 538.5 were all showed in Fig. 1 . The potassium ion derived from human blood can combine with both of ertapenem and the hydrolysate and form peaks such as $[\mathrm{M}+\mathrm{K}]^{+},\left[\mathrm{M}_{\mathrm{hydr} / \text { decarb }}+\mathrm{K}\right]^{+}$, etc. These peaks were analyzed when mass spectrums of ertapenem solutions incubated with carbapenemase producers or non-carbapenemase producers were imported in Flexanalysis 3.0 software. Carbapenemase producers can be distinguished preliminarily by the following criteria: (1) almost absence of all ertapenem intact peaks; (2) presence of peaks corresponding to hydrolysed, decarboxylated form with high intensity.

\section{LogRQ value calculated with the intensity of peaks}

As a matter of fact, the subjective judgment of carbapenemase and non-carbapenemase producers according to the artificial observation of these peaks was ambiguous. We introduced $\log \mathrm{RQ}$ value to analyze the intensities of these peaks. After $2 \mathrm{~h}$ incubation with ertapenem solution, the average of $\log R Q$ value of 32 non-carbapenemase producers were $-0.85 \pm 0.14$, and 32 carbapenemase producers were $0.87 \pm 0.55$ (Fig. 2).

\section{Cutoff value of logRQ}

The receiver operating characteristic (ROC) curve of the method with $\log R Q$ values was drawn in Fig. 3 . The ROC analysis results showed that the $\log R Q$ value was effective in distinguishing carbapenemase producers and noncarbapenemase producers (area under curve, $\mathrm{AUC}=1, \mathrm{P}$ value $<0.001)$. These two groups were discriminated by the cutoff value of $\log R Q-0.45$ with a sensitivity of $100 \%$ and specificity of $100 \%$.

\section{Efficiency of MALDI-TOF MS based hydrolysis assay}

A total of 385 clinical blood culture bottles contained Enterobacteriaceae strains from different body parts of 218 patients were collected. $81.3 \%$ (313/385) were sensitive to carbapenem, including Escherichia coli (207), Klebsiella pneumoniae (68), Enterobacter cloacae (15), Klebsiella oxytoca (6), Serratia marcescens (5), Citrobacter freundii (3), Citrobacter koseri (3), Enterobacter aerogenes (2), Morganella morganii (2), Proteus mirabilis (2). The $\log R Q$ values of all 313 isolates were $<-0.45$ with the accuracy rate of $100 \%$. And $18.7 \%$ (72/385) of clinical blood culture bottles contained CRE (Table 1). The $\operatorname{logRQ}$ values of $62 \mathrm{CRE}$ were $\geqq-0.45$, while the other 10 CRE were $<-0.45$. Comparing with the detection of carbapenemase genes, MALDI-TOF MS based ertapenem hydrolysis assay of carbapenemase detection has a sensitivity of 92.5\% (62/67) [95\% CI 86.2-98.8\%] and specificity of $100 \%(5 / 5)$ [95\% CI 100-100\%].

\section{Discussion}

MALDI-TOF MS has been used commonly for direct identification of microorganisms from positive blood cultures in clinical microbiology laboratories. There are various bacterial extraction methods for direct blood culture identification, including blood cells lysis [12-16], differential velocity centrifugation [17] and serum separator tubes usage [18]. Nowadays, MALDI-TOF MS has not only been used for identification of pathogens but 


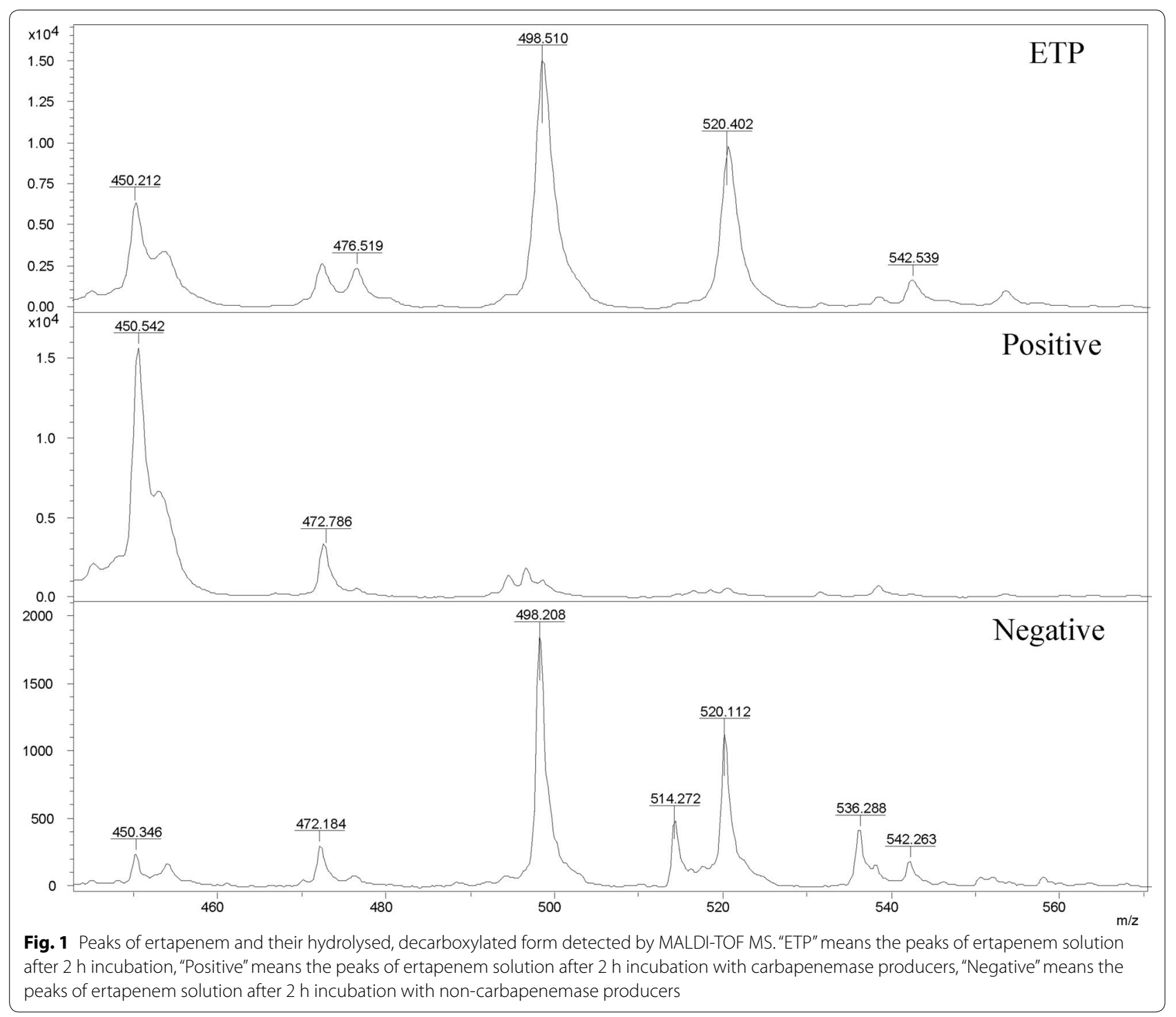

also for detection of antimicrobial resistance [19, 20]. In our laboratory, serum separator tubes were used to collect bacterial pellets. After placing the pellets onto the target plate, MALDI-TOF MS was directly performed for identification. This protocol was routinely performed for positive blood cultures in our laboratory. The species identification reports of positive blood cultures usually delivered to clinicians within $30 \mathrm{~min}$. In our previous research, the carbapenemase activity of Enterobacteriaceae strains that growed on Columbia blood agar was detected rapidly and accurately by MALDI-TOF MS based ertapenem hydrolysis assay. Based on that, the carbapenemase activity of Enterobacteriaceae strains in positive blood cultures collected from clinical patients was detected by the same assay. Different from the other researches, only simulated blood cultures were chosen here as the objects $[9,21,22]$. We first established a blood culture simulation method and calculated the cutoff values of $\log R \mathrm{Q}$. According to the $\log R \mathrm{Q}$ values, we test the real positive blood cultures from patients to evaluate the efficiency of this method. The results revealed that this MALDI-TOF MS based ertapenem hydrolysis assay showed more clinical significance than before.

In our study, various species of Enterobacteriaceae strains which contained different types of carbapenemase genes were selected for positive blood culture simulations. These strains almost covered all the types of species and carbapenemase genes that could be detected in clinical microbiology laboratory. Therefore, MALDI-TOF MS based hydrolysis assay here is suitable to detect the carbapenemase activity of all species of the Enterobacteriaceae strains in blood cultures. 

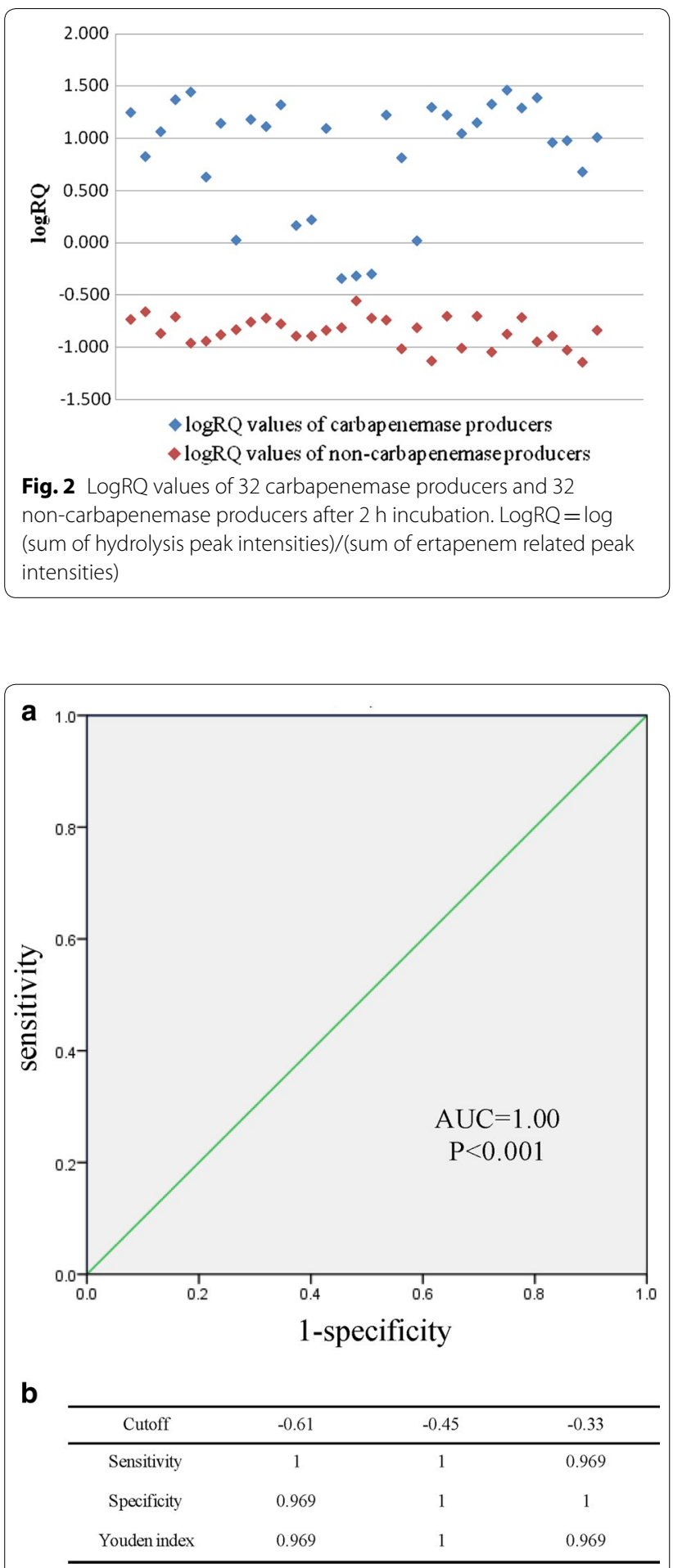

Fig. 3 Statistical analysis with the logRQ values of the blood culture simulation. a ROC curves of the method that using logRQ values to identify carbapenemase producers. b Sensitivity, specificity and youden index with different cutoff values of logRQ
The limitation of MALDI-TOF MS based hydrolysis assay is that this method could only be used to detect carbapenemase activity which is one of the most important drug resistant mechanisms of CRE. In our study, 5 CRE strains were identified as non-carbapenemase producer by MALDI-TOF MS based hydrolysis assay. Besides that, no carbapenemase genes which mentioned in Materials and Methods were detected in these 5 isolates. The reason may be the presence of other carbapenem resistance mechanisms [23]. 41.7\% (5/12) NDM-1 carbapenemase gene-positive Enterobacteriaceae isolates were distinguished as non-carbapenemase producer with the $\log \mathrm{RQ}$ values of below -0.45 . However, as reported before, carbapenemase-producing Enterobacteriaceae isolates which carried NDM-1 can hydrolyse carbapenem completely after $2 \mathrm{~h}$ incubation [21, 22, 24-26]. Pure colonies other than strains collected from blood cultures were taken as research objects in most of these researches. Compared with pure colonies grown on blood agar, the amount of bacteria in pellets collected from positive blood cultures is not easily detected which will lead to the false-negative results. In our study, there were several clinical blood culture bottles which contained two species of Enterobacteriaceae strains. These strains in the same blood culture bottles which all showed sensitive to carbapenem were identified as non-carbapenemase producers by MALDI-TOF MS based hydrolysis assay. Besides, as long as there was one strain in the blood culture bottles showed resistant to carbapenem, all these two strains were identified as carbapenemase producers. The results showed that there were no needs to separate the multi-microorganisms in positive blood cultures when detected carbapenemase activity by MALDI-TOF MS. This method can help to evaluate the effectiveness of carbapenem treatment timely.

The antibiotic sensitivity reports were essential for clinical treatment of patients whose blood cultures were determined as positive. At our laboratory, MALDI-TOF MS has been routinely used to directly identify the species of strains from positive blood cultures. Our data showed that MALDI-TOF MS based ertapenem hydrolysis assay can directly detect carbapenemase of Enterobacteriaceae strains isolated from blood cultures with high sensitivity and specificity. Thus, when the assay is routinely performed for positive blood cultures, we could deliver the reports, especially positive results, to the clinicians within $3 \mathrm{~h}$ and provide them evidence to choose appropriate combined medication regimen for the patient early. 
Table 1 The results of ETP hydrolysis assay and carbapenemase gene detection of CRE strains isolated from clinical blood cultures

\begin{tabular}{|c|c|c|c|}
\hline Bacterial species (n) & Carbapenemase genes (n) & LogRQ value $\geqq-0.45$ & $\begin{array}{l}\log R Q \\
\text { value }<-0.45\end{array}$ \\
\hline \multirow[t]{3}{*}{ Klebsiella pneumoniae (64) } & KPC-2 (54) & 54 & 0 \\
\hline & NDM-1 (5) & 3 & 2 \\
\hline & $\operatorname{Neg}(5)$ & 0 & 5 \\
\hline Escherichia coli (2) & NDM-1(2) & 2 & 0 \\
\hline Klebsiella oxytoca $(2)$ & NDM-1 (2) & 2 & 0 \\
\hline Enterobacter aerogenes (2) & NDM-1 (2) & 0 & 2 \\
\hline Citrobacter freundii (1) & KPC-2 and IMP-4 ${ }^{\mathrm{a}}(1)$ & 1 & 0 \\
\hline Morganella morganii (1) & NDM-1 (1) & 0 & 1 \\
\hline Total (72) & & 62 & 10 \\
\hline
\end{tabular}

'Neg' means no carbapenemase genes detected

a The isolates that contained both $b / a_{K P C-2}$ and $b / a_{I M P-4}$ carbapenemase genes

\section{Conclusions}

MALDI-TOF MS based ertapenem hydrolysis assay is a rapid, accurate, effective and economic method to detect carbapenemase activity of Enterobacteriaceae strains isolated from positive blood cultures. With the high specificity and sensitivity, this method can be used as routine protocol to detect carbapenemase in clinical workflows. So clinicians can acquire meaningful reference early to treat bloodstream infection caused by Enterobacteriaceae strains.

\begin{abstract}
Abbreviations
MALDI-TOF MS: matrix-assisted laser desorption ionization time-of-flight mass spectrometry; CRE: carbapenemase-resistant Enterobacteriaceae; ETP: ertapenem ; KPC: Klebsiella pneumoniae Carbapenemase; NDM: New-DelhiMetallo-betalactamase; IMP: Imipenemase; VIM: verona metallo- $\beta$-lactamase; OXA: Oxacillinase.
\end{abstract}

\section{Authors' contributions}

Ying Liu, Lisong Shen and Jiajia Yu proposed and designed the study. Jiajia Yu collected, analysed and interpreted the data. Jing Yu, Jingxian Liu, Weinan Zhu and Yuanrui Li participated in proofreading of the manuscript and in critical revision. All authors read and approved the final manuscript.

\section{Acknowledgements}

Not applicable.

\section{Competing interests}

The authors declare that they have no competing interests.

\section{Availability of data and materials}

All data generated or analysed during this study are included in this published article.

\section{Consent for publication}

Not applicable.

Ethics approval and consent to participate

Not applicable.

\section{Funding}

This study was supported by a grant from Shanghai Shenkang Hospital Development Center and Shanghai Health Bureau. The content of this article is solely our responsibility and does not represent any institute's official views.

\section{Publisher's Note}

Springer Nature remains neutral with regard to jurisdictional claims in published maps and institutional affiliations.

Received: 30 August 2017 Accepted: 5 May 2018

Published online: 18 May 2018

\section{References}

1. Kumar A, Roberts D, Wood KE, Light B, Parrillo JE, Sharma S, Suppes R, Feinstein D, Zanotti S, Taiberg L, et al. Duration of hypotension before initiation of effective antimicrobial therapy is the critical determinant of survival in human septic shock. Crit Care Med. 2006;34(6):1589-96.

2. Kumar A, Ellis P, Arabi Y, Roberts D, Light B, Parrillo JE, Dodek P, Wood G, Simon D, Peters C, et al. Initiation of inappropriate antimicrobial therapy results in a fivefold reduction of survival in human septic shock. Chest. 2009;136(5):1237-48.

3. Trenholme GM, Kaplan RL, Karakusis PH, Stine T, Fuhrer J, Landau W, Levin S. Clinical impact of rapid identification and susceptibility testing of bacterial blood culture isolates. J Clin Microbiol. 1989;27(6):1342-5.

4. Tariq TM, Rasool E. Emerging trends of bloodstream infections: a six-year study at a paediatric tertiary care hospital in Kabul. J Coll Physicians Surg Pak JCPSP. 2016;26(11):887-91.

5. Hsu LY, Apisarnthanarak A, Khan E, Suwantarat N, Ghafur A, Tambyah PA. Carbapenem-resistant Acinetobacter baumannii and Enterobacteriaceae in south and southeast Asia. Clin Microbiol Rev. 2017;30(1):1-22.

6. Bassetti M, Carnelutti A, Peghin M. Patient specific risk stratification for antimicrobial resistance and possible treatment strategies in Gram-negative bacterial infections. Expert Rev Anti Infect Ther. 2017;15(1):55-65.

7. Hu FP, Guo Y, Zhu DM, Wang F, Jiang XF, Xu YC, Zhang XJ, Zhang CX, Ji P, Xie $Y$, et al. Resistance trends among clinical isolates in China reported from CHINET surveillance of bacterial resistance, 2005-2014. Clin Microbiol Infect. 2016;22(Suppl 1):S9-14.

8. Tumbarello M, Trecarichi EM, De Rosa FG, Giannella M, Giacobbe DR, Bassetti M, Losito AR, Bartoletti M, Del Bono V, Corcione S, et al. Infections caused by KPC-producing Klebsiella pneumoniae: differences in therapy and mortality in a multicentre study. J Antimicrob Chemother. 2015;70(7):2133-43. 
9. Johansson A, Nagy E, Soki J. Instant screening and verification of carbapenemase activity in Bacteroides fragilis in positive blood culture, using matrix-assisted laser desorption ionization-time of flight mass spectrometry. J Med Microbiol. 2014;63(Pt 8):1 105-10.

10. Jung JS, Popp C, Sparbier K, Lange C, Kostrzewa M, Schubert S. Evaluation of matrix-assisted laser desorption ionization-time of flight mass spectrometry for rapid detection of beta-lactam resistance in Enterobacteriaceae derived from blood cultures. J Clin Microbiol. 2014;52(3):924-30.

11. Liu JX, Yu J, Li YR, Liu Y. Study on resistance mechanism of Klebsiella pneumoniae towards carbapenem. J Shanghai Jiaotong Univ (Med Sci). 2016;36(1):93-9.

12. Randazzo A, Simon M, Goffinet P, Classen JF, Hougardy N, Pierre P, Kinzinger P, Mauel E, Goffinet JS. Optimal turnaround time for direct identification of microorganisms by mass spectrometry in blood culture. J Microbiol Methods. 2016;130:1-5.

13. Wattal C, Oberoi JK. Microbial identification and automated antibiotic susceptibility testing directly from positive blood cultures using MALDITOF MS and VITEK 2. Eur J Clin Microbiol Infect Dis. 2016;35(1):75-82.

14. Verroken A, Defourny L, le Polain de Waroux O, Belkhir L, Laterre PF, Delmee M, Glupczynski Y. Clinical impact of MALDI-TOF MS identification and rapid susceptibility testing on adequate antimicrobial treatment in sepsis with positive blood cultures. PLoS ONE. 2016;11(5):e0156299.

15. Thomin J, Aubin GG, Foubert F, Corvec S. Assessment of four protocols for rapid bacterial identification from positive blood culture pellets by matrix-assisted laser desorption ionization-time of flight mass spectrometry (Vitek(R) MS). J Microbiol Methods. 2015;115:54-6.

16. Lagace-Wiens P. Matrix-assisted laser desorption/ionization time of flight mass spectrometry (MALDI-TOF/MS)-based identification of pathogens from positive blood culture bottles. Methods Mol Biol (Clifton, NJ). 2015;1237:47-55.

17. Angeletti S, Dicuonzo G, D'Agostino A, Avola A, Crea F, Palazzo C, Dedej E, De Florio L. Turnaround time of positive blood cultures after the introduction of matrix-assisted laser desorption-ionization time-of-flight mass spectrometry. New Microbiol. 2015;38(3):379-86.

18. Barnini S, Brucculeri V, Morici P, Ghelardi E, Florio W, Lupetti A. A new rapid method for direct antimicrobial susceptibility testing of bacteria from positive blood cultures. BMC Microbiol. 2016;16(1):185.
19. Hart PJ, Wey E, McHugh TD, Balakrishnan I, Belgacem O. A method for the detection of antibiotic resistance markers in clinical strains of Escherichia coli using MALDI mass spectrometry. J Microbiol Methods. 2015;111:1-8,

20. Monteferrante CG, Sultan S, Ten Kate MT, Dekker LJ, Sparbier K, Peer M, Kostzrewa M, Luider TM, Goessens WH, Burgers PC. Evaluation of different pretreatment protocols to detect accurately clinical carbapenemaseproducing Enterobacteriaceae by MALDI-TOF. J Antimicrob Chemother. 2016;71(10):2856-67.

21. Ghebremedhin B, Halstenbach A, Smiljanic M, Kaase M, Ahmad-Nejad P. MALDI-TOF MS based carbapenemase detection from culture isolates and from positive blood culture vials. Ann Clin Microbiol Antimicrob. 2016;15(1):5

22. Hoyos-Mallecot Y, Riazzo C, Miranda-Casas C, Rojo-Martin MD, GutierrezFernandez J, Navarro-Mari JM. Rapid detection and identification of strains carrying carbapenemases directly from positive blood cultures using MALDI-TOF MS. J Microbiol Methods. 2014;105:98-101.

23. Doumith M, Ellington MJ, Livermore DM, Woodford N. Molecular mechanisms disrupting porin expression in ertapenem-resistant Klebsiella and Enterobacter spp. clinical isolates from the UK. J Antimicrob Chemother. 2009;63(4):659-67.

24. Hoyos-Mallecot Y, Cabrera-Alvargonzalez JJ, Miranda-Casas C, Rojo-Martin MD, Liebana-Martos C, Navarro-Mari JM. MALDI-TOF MS, a useful instrument for differentiating metallo-beta-lactamases in Enterobacteriaceae and Pseudomonas spp. Lett Appl Microbiol. 2014;58(4):325-9.

25. Lee W, Chung HS, Lee Y, Yong D, Jeong SH, Lee K, Chong Y. Comparison of matrix-assisted laser desorption ionization-time-of-flight mass spectrometry assay with conventional methods for detection of IMP-6, VIM-2, NDM-1, SIM-1, KPC-1, OXA-23, and OXA-51 carbapenemase-producing Acinetobacter spp., Pseudomonas aeruginosa, and Klebsiella pneumoniae. Diagn Microbiol Infect Dis. 2013;77(3):227-30.

26. Hrabák J, Študentová V, Walková R, Žemličková H, Jakubů V, Chudáčková E, Gniadkowski M, Pfeifer Y, Perry JD, Wilkinson K, et al. Detection of NDM1, VIM-1, KPC, OXA-48, and OXA-162 carbapenemases by matrix-assisted laser desorption ionization-time of flight mass spectrometry. J Clin Microbiol. 2012;50(7):2441-3.

Ready to submit your research? Choose BMC and benefit from

- fast, convenient online submission

- thorough peer review by experienced researchers in your field

- rapid publication on acceptance

- support for research data, including large and complex data types

- gold Open Access which fosters wider collaboration and increased citations

- maximum visibility for your research: over 100M website views per year

At BMC, research is always in progress.

Learn more biomedcentral.com/submissions 